\title{
Effect of stress-dosed hydrocortisone on physical capacity in patients with Addison's disease (AD)
}

Katerina Simunkova ${ }^{1}$, Nevena Jovanovic ${ }^{2}$, Espen Rostrup ${ }^{1}$, Paal Methlie ${ }^{2}$, Marianne $\varnothing_{\text {ksnes }}^{2}{ }^{2}$, Roy Miodini Nilsen ${ }^{3}$, Hanne Henn ${ }^{1}$, Mira Tilseth ${ }^{1}$, Kristin Godang ${ }^{4}$, Ana

Kovac $^{1}$, Kristian Løvås ${ }^{1,2}$ and Eystein S Husebye ${ }^{1,2}$

Departments of 1 Clinical Science and 3Global Public Health and Primary Care University of Bergen, 4021 BERGEN, Norway. 2Department of Medicine, Haukeland University Hospital, 5021 BERGEN, Norway.4Department of Endocrinology, Oslo University Hospital Rikshospitalet, Sognsvannsveien 20, 0372 Oslo, Norway

Aim: To evaluate the effect of stress dose hydrocortisone $(\mathrm{HC})$ on physical activity in female patients with AD.

Introduction: Many patients take stress doses during physical or psychological events and report benefit on performance and postexertion fatigue. The effect of such dosing has not been demonstrated.

\section{Results:}

- V02max and duration of exercise were lower in AD than controls, and did not improve with stress dosing.

- The glucose response to exercise was attenuated in the patients compared with the controls

- The adrenaline response to exercise was flattened in patients compared with controls.

\begin{tabular}{|c|c|c|c|c|c|c|}
\hline \multirow[b]{2}{*}{ Parameters } & Treatment & \multicolumn{2}{|c|}{ Placebo } & \multicolumn{2}{|c|}{ Healthy subjects } & \multirow[b]{2}{*}{$\begin{array}{r}P \text { for } \\
\text { interaction }\end{array}$} \\
\hline & $\begin{array}{l}\text { Observed } \\
\text { Mean } \pm \text { SE }\end{array}$ & $\begin{array}{l}\text { Observed } \\
\text { Mean } \pm \text { SE }\end{array}$ & $\begin{array}{l}\text { Predicted Mean } \\
\text { Difference }(95 \% \\
\mathrm{CI})^{\mathrm{s}}\end{array}$ & $\begin{array}{r}\text { Observed } \\
\text { Mean } \pm \text { SE }\end{array}$ & $\begin{array}{r}\text { Predicted Mean } \\
\text { Difference }(95 \% \mathrm{CI})^{\mathrm{a}}\end{array}$ & \\
\hline \multirow{2}{*}{\multicolumn{7}{|c|}{ FFA $^{d}$}} \\
\hline & & & & & & $0.7 \mathrm{f} / 0.002 \mathrm{~g}$ \\
\hline Before & $0.4 \pm 0.05$ & $0.4 \pm 0.07$ & $0.03(-0.10,0.16)$ & $0.4 \pm 0.08$ & $0.03(-0.21,0.27)$ & \\
\hline After & $0.3 \pm 0.04$ & $0.3 \pm 0.04$ & $-0.01(-0.15,0.12)$ & $0.4 \pm 0.06$ & $0.03(-0.21,0.27)$ & \\
\hline $15 \mathrm{~min}$ & $0.4 \pm 0.05$ & $0.4 \pm 0.07$ & $-0.01(-0.14,0.12)$ & $0.3 \pm 0.06$ & $-0.07(-0.31,0.17)$ & \\
\hline $30 \mathrm{~min}$ & $0.4 \pm 0.05$ & $0.4 \pm 0.06$ & $-0.03(-0.16,0.09)$ & $0.3 \pm 0.05$ & $-0.12(-0.37,0.12)$ & \\
\hline \multirow{2}{*}{\multicolumn{7}{|c|}{$\begin{array}{l}\text { Glucoser } \\
(\mathrm{mmol} / \mathrm{L})\end{array}$}} \\
\hline & & & & & & 0.06 \\
\hline Before & $5.0 \pm 0.1$ & $4.7 \pm 0.1$ & $-0.29(-0.68,0.10)$ & $5.4 \pm 0.2$ & $0.36(-0.46,1.18)$ & \\
\hline After & $5.0 \pm 0.1$ & $5.1 \pm 0.1$ & $0.04(-0.37,0.46)$ & $6.0 \pm 0.3$ & $1.00(0.17,1.83)$ & \\
\hline $15 \mathrm{~min}$ & $5.3 \pm 0.1$ & $5.1 \pm 0.1$ & $-0.17(-0.56,0.22)$ & $5.9 \pm 0.3$ & $0.69(-0.14,1.51)$ & \\
\hline $30 \min$ & $5.1 \pm 0.1$ & $4.9 \pm 0.1$ & $-0.18(-0.57,0.21)$ & $5.7 \pm 0.3$ & $0.48(-0.35,1.31)$ & \\
\hline \multicolumn{7}{|l|}{ Insuline } \\
\hline$(\mathrm{mIE} / \mathrm{L})$ & & & & & & 0.34 \\
\hline Before & $7.05 \pm 2.11$ & $7.26 \pm 1.53$ & $0.21(-3.67,4.09)$ & $9.77 \pm 2.44$ & $2.72(-4.89,10.33)$ & \\
\hline After & $7.14 \pm 2.13$ & $8.92 \pm 1.95$ & $0.70(-3.42,4.83)$ & $14.51 \pm 1.56$ & $6.60(-1.07,14.28)$ & \\
\hline $15 \mathrm{~min}$ & $11.06 \pm 2.88$ & $10.97 \pm 2.12$ & $-0.09(-3.97,3.79)$ & $14.01 \pm 1.34$ & $2.59(-4.66,10.57)$ & \\
\hline $30 \mathrm{~min}$ & $9.73 \pm 2.54$ & $9.18 \pm 2.06$ & $-0.55(-4.43,3.33)$ & $11.97 \pm 1.60$ & $2.29(-5.39,9.98)$ & \\
\hline $\mathrm{GH}^{\mathrm{e}}(\mathrm{ug} / \mathrm{L})$ & & & & & & $0.8^{\prime} / 0.3 \mathrm{~g}$ \\
\hline Before & $-0.62 \pm 0.61$ & $-0.79 \pm 0.57$ & $-0.49(-2.62,1.64)$ & $0.30 \pm 0.37$ & $0.69(-2.15,3.53)$ & \\
\hline After & $-0.19 \pm 0.71$ & $0.20 \pm 0.52$ & $0.44(-1.83,2.70)$ & $0.50 \pm 0.37$ & $0.14(-2.74,3.03)$ & \\
\hline $15 \mathrm{~min}$ & $0.47 \pm 0.47$ & $0.43 \pm 0.40$ & $-0.47(-2.60,1.66)$ & $0.48 \pm 0.31$ & $-0.86(-3.70,1.98)$ & \\
\hline $30 \mathrm{~min}$ & $0.37 \pm 0.42$ & $0.37 \pm 0.42$ & $-0.13(-2.26,2.00)$ & $0.05 \pm 0.37$ & $-1.03(-3.92,1.85)$ & \\
\hline \multicolumn{7}{|l|}{ Lactate } \\
\hline$(\mathrm{mmol} / \mathrm{L})$ & & & & & & 0.81 \\
\hline Before & $1.07 \pm 0.24$ & $1.12 \pm 0.13$ & $0.05(-1.71,1.82)$ & $0.98 \pm 0.10$ & $-0.09(-2.06,1.88)$ & \\
\hline After & $7.05 \pm 0.79$ & $6.48 \pm 0.67$ & $-0.57(-2.34,1.99)$ & $6.51 \pm 0.51$ & $-0.54(-2.51,1.43)$ & \\
\hline $15 \mathrm{~min}$ & $4.40 \pm 0.63$ & $4.06 \pm 0.61$ & $-0.34(-2.11,1.43)$ & $4.67 \pm 0.59$ & $0.24(-1.70,2.24)$ & \\
\hline $30 \mathrm{~min}$ & $2.19 \pm 0.34$ & $2.09 \pm 0.47$ & $-0.10(-1.87,1.67)$ & $2.91 \pm 0.53$ & $0.75(-1.27,2.22)$ & \\
\hline
\end{tabular}

Table 1. Comparison of selected parameters among patients and healthy subjects a linear mixed effect models with random intercept; $95 \% \mathrm{CI}$ for difference by post-hoc test for pairwise comparison

$b$ Overall P for interaction by likelihood ratio test.

$b$ Overall P for interaction by likelihood ratio test.
$c$ No statistically significant period or sequence effects.

$d$ A statistically significant period effect for the cross-over of the treatment and placebo.

$e$ A statistically significant sequence effect for the cross-over of the treatment and placebo.

separate analysis for treatment and placebo.

$g$ separate analyis for treatment and controls.

\begin{tabular}{|c|c|c|c|c|c|c|}
\hline \multirow[b]{3}{*}{ Parameters } & \multirow{3}{*}{$\begin{array}{c}\text { Treatment } \\
\text { Observed } \\
\text { Mean } \pm \text { SE }\end{array}$} & \multicolumn{2}{|c|}{ Placebo } & \multicolumn{2}{|c|}{ Healthy subjects } & \multirow{3}{*}{$\begin{array}{c}\text { P for } \\
\text { difference }\end{array}$} \\
\hline & & Observed & $\begin{array}{l}\text { Predicted Mean } \\
\text { Difference ( } 95 \%\end{array}$ & Observed & Predicted Mean & \\
\hline & & Mean $\pm S E$ & $\mathrm{CI})^{2}$ & Mean $\pm S E$ & Difference $(95 \% \mathrm{CI})^{2}$ & \\
\hline $\operatorname{Load}_{\max }(\mathrm{W})$ & $141 \pm 32.1$ & $142 \pm 33$ & $0.8(-4.40,5.96)$ & $186 \pm 35$ & $44.7(12.51,76.87)$ & 0.02 \\
\hline $\mathrm{Time}_{\max }(\mathrm{sec})$ & $344 \pm 108$ & $353 \pm 99.7$ & $9.0(9.82,27.82)$ & $490 \pm 98$ & $146.0(50.05,241.95)$ & 0.007 \\
\hline $\mathrm{vO}_{2 \max }(\mathrm{L} / \mathrm{min})$ & $1.56 \pm 0.43$ & $17.5 \pm 50.1$ & $0.13(-0.063,0.33)$ & $2.33 \pm 0.55$ & $0.77(0.31,1.23)$ & 0.003 \\
\hline $\mathrm{VO}_{2} \mathrm{~kg}_{\max }(\mathrm{ml} / \mathrm{kg} / \mathrm{min})$ & $25.7 \pm 8.37$ & $26.6 \pm 8.11$ & $0.91(-2.10,3.912)$ & $34.1 \pm 7.35$ & $8.49(1.04,15.93)$ & 0.06 \\
\hline $\mathrm{VCO}_{2 \max }(\mathrm{L} / \mathrm{min})$ & $1.9 \pm 0.489$ & $2.03 \pm 0.49$ & $0.16(-0.08,0.35)$ & $2.73 \pm 0.66$ & $0.83(0.28,1.38)$ & 0.007 \\
\hline $\mathrm{RER}_{\max }$ & $1.2 \pm 0.046$ & $1.21 \pm 0.046$ & $0.01(-0.02,0.04)$ & $1.17 \pm 0.054$ & $-0.04(-0.08,0.01)$ & 0.1 \\
\hline $\mathrm{BPdia}_{\max }(\mathrm{mmHg})$ & $94.2 \pm 8.59$ & $94.7 \pm 11.8$ & $0.5(-3.96,4.96)$ & $95 \pm 13.4$ & $0.80(-10.44,12.04)$ & 0.9 \\
\hline $\mathrm{BP}_{\mathrm{sys}_{\max }}(\mathrm{mmHg})$ & $186 \pm 19.2$ & $192 \pm 18.9$ & $6.0(-3.25,15.25)$ & $195 \pm 26$ & $9.2(-12.05,30.45)$ & 0.3 \\
\hline $\mathrm{HR}_{\max }$ (beats/min) & $160 \pm 20.3$ & $159 \pm 15.6$ & $-0.9(-9.08,7.28)$ & $172 \pm 12$ & $11.8(-3.13,26.73)$ & 0.2 \\
\hline $\mathrm{O}_{2} \mathrm{pul}_{\max }(\mathrm{ml})$ & $9.82 \pm 1.84$ & $10.7 \pm 2.16$ & $0.91(-0.37,2.19)$ & $13.5 \pm 3.22$ & $3.71(1.24,6.18)$ & 0.007 \\
\hline Recovery (sec) & $53 \pm 24.1$ & $58 \pm 22$ & $5.0(-7.63,17.63)$ & $107 \pm 32.3$ & $54.0(27.86,80.14)$ & 0.001 \\
\hline
\end{tabular}

\section{Table 2.Physical and cardiorespiratory parameters in response to a cycle test} a. By linear mixed effect models with random intercept; $95 \%$ CI for difference was obtained by post-hoc test for pairwise comparison (Sidak corrected). b. Overall P value for group difference obtained by likelihood ratio test.

Loadmax - Load max, timemax- duration of exercise, V02max -oxygen uptake, V02kgmax oxygen uptake per $\mathrm{kg}$, VC02max- carbon dioxide production, RERmax- respiratory exchange rate, BPdiamax- Peak diastolic blood preassure, BPsysmax-Peak systolic blood preassure, HRmax- Peak heart rate, O2pulsmax-maximum peak of oxygen per pulse, Eemax- Energetic expenditure max, METSmax -Metabolic equivalents max, Recover-Time of recovery.

\section{Conclusions}

- Stress dosing does not seem warranted for short-term exercise

- Dysfunction of the adrenal medulla and impaired glucose response to stress might lower performance and increase post-exertion fatigue in AD

\section{Design}

Double blind, controlled, cross-over designed, randomized pilot trial to investigate the effects of $\mathbf{1 0} \mathbf{~} \mathbf{~ g}$ oral $\mathbf{H C}$ on ergometer test to exhaustion.

\section{Participants}

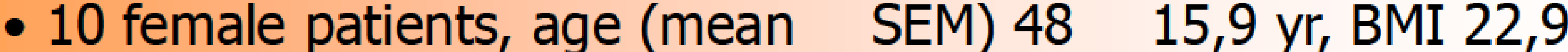
$4,6 \mathrm{~kg}$. Regular treatment was cortisone acetate $30.17 .6 \mathrm{mg}$ (range 18.7-37.5 mg) and fludrocortisone $0.095 \quad 0.015 \mathrm{mg}$ (0.05-0.1 mg).

- 10 age and BMI-matched healthy female controls.

\section{Outcomes}

- primary endpoint: oxygen uptake (O2 uptake) and maximal aerobic capacity $\left(\mathrm{V}_{\mathrm{O} 2} \mathrm{max}\right)$

- secondary endpoints: detailed cardiorespiratory parameters, duration of exercise, post-exercise hypoglycaemic events and glycaemic variability, endocrine and metabolic responses, and HRQoL evaluated by questionnaires.
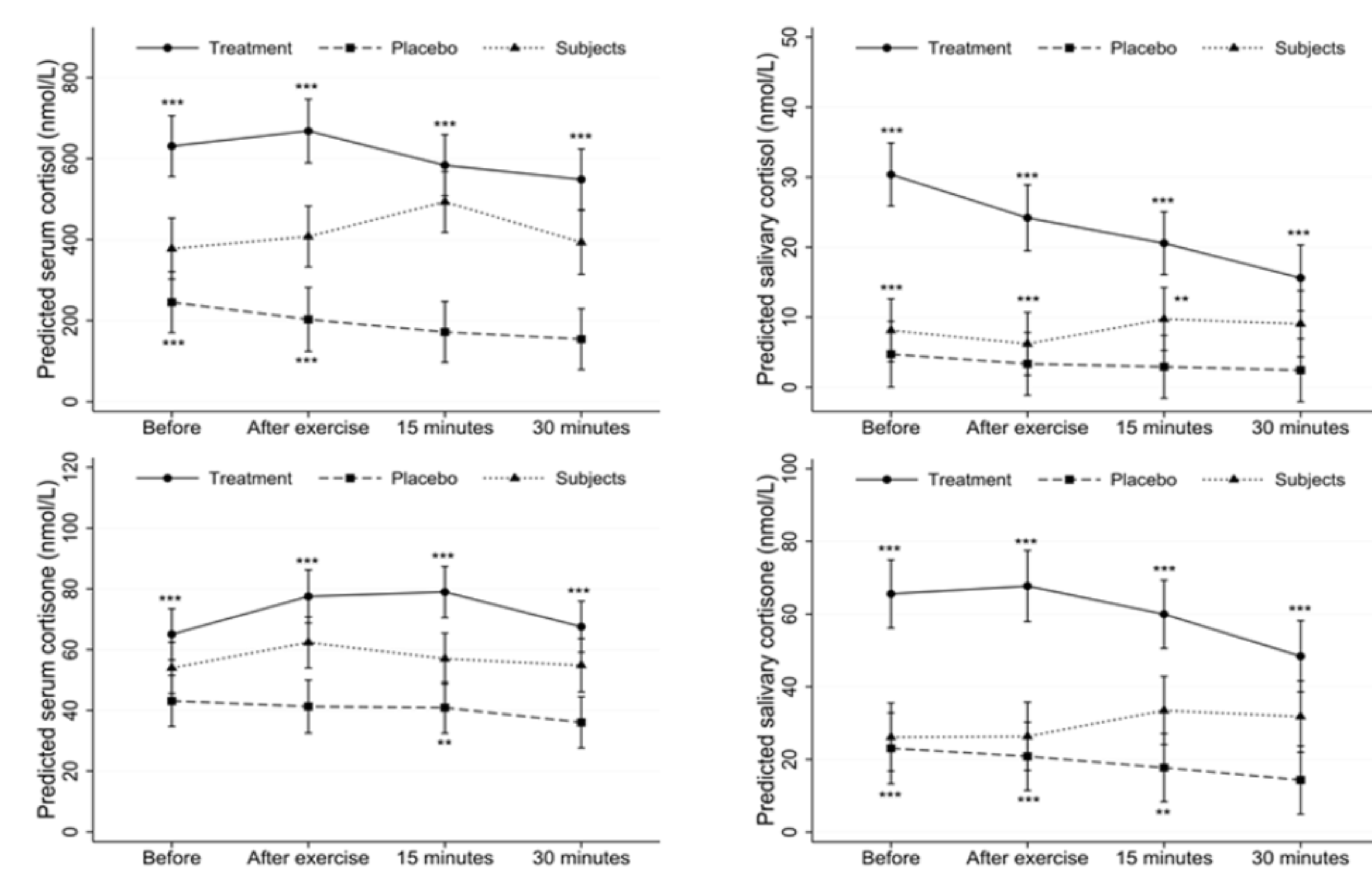

Figure 1. Serum or salivary cortisol, cortisone in patients and healthy subjects.

${ }^{* * * \mathrm{P}} \leq 0.001$ for treatment vs placebo, $* * * \mathrm{P} \leq 0.001$ for treatment vs subjects, $* * \mathrm{P} \leq 0.01$ for treatment vs subjects
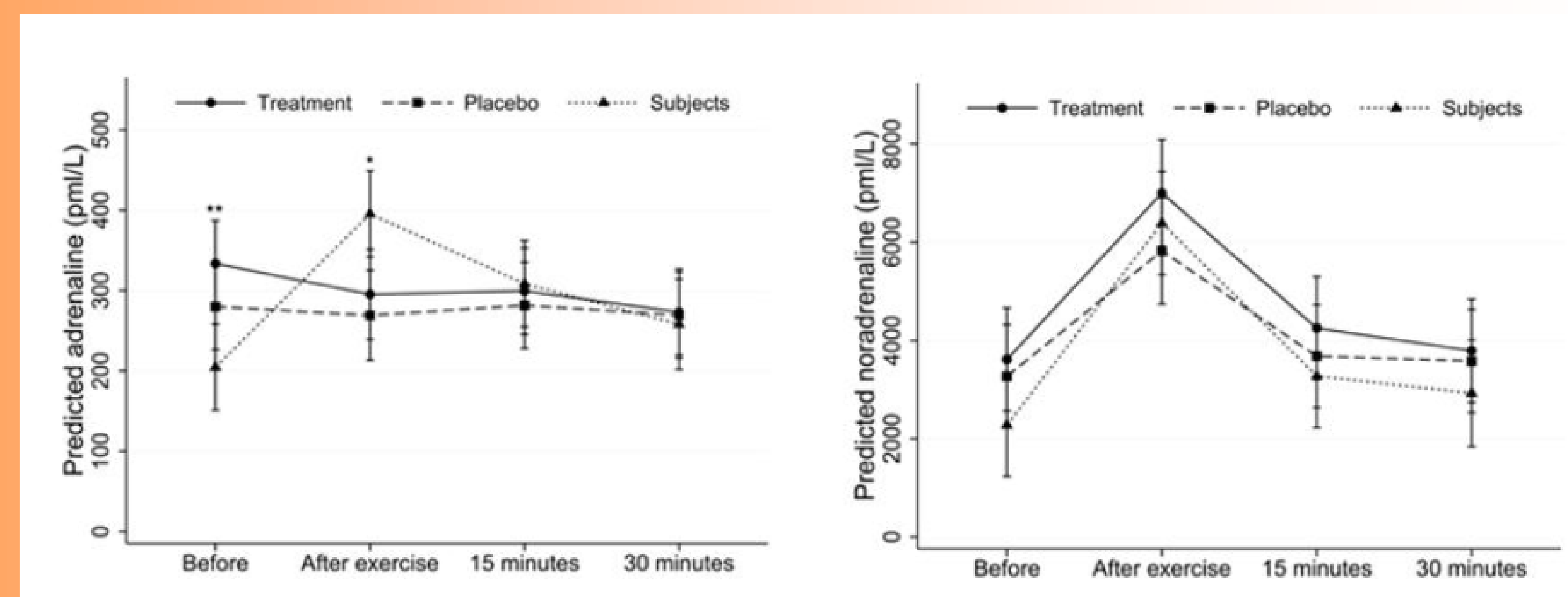

Figure 2. Levels of plasma adrenaline and noradrenaline in patients and healthy subjects ${ }^{*} \mathrm{P} \leq 0.001$ for treatment vs healthy subjects $* \mathrm{P} \leq 0.01$ for treatment vs healthy subjects

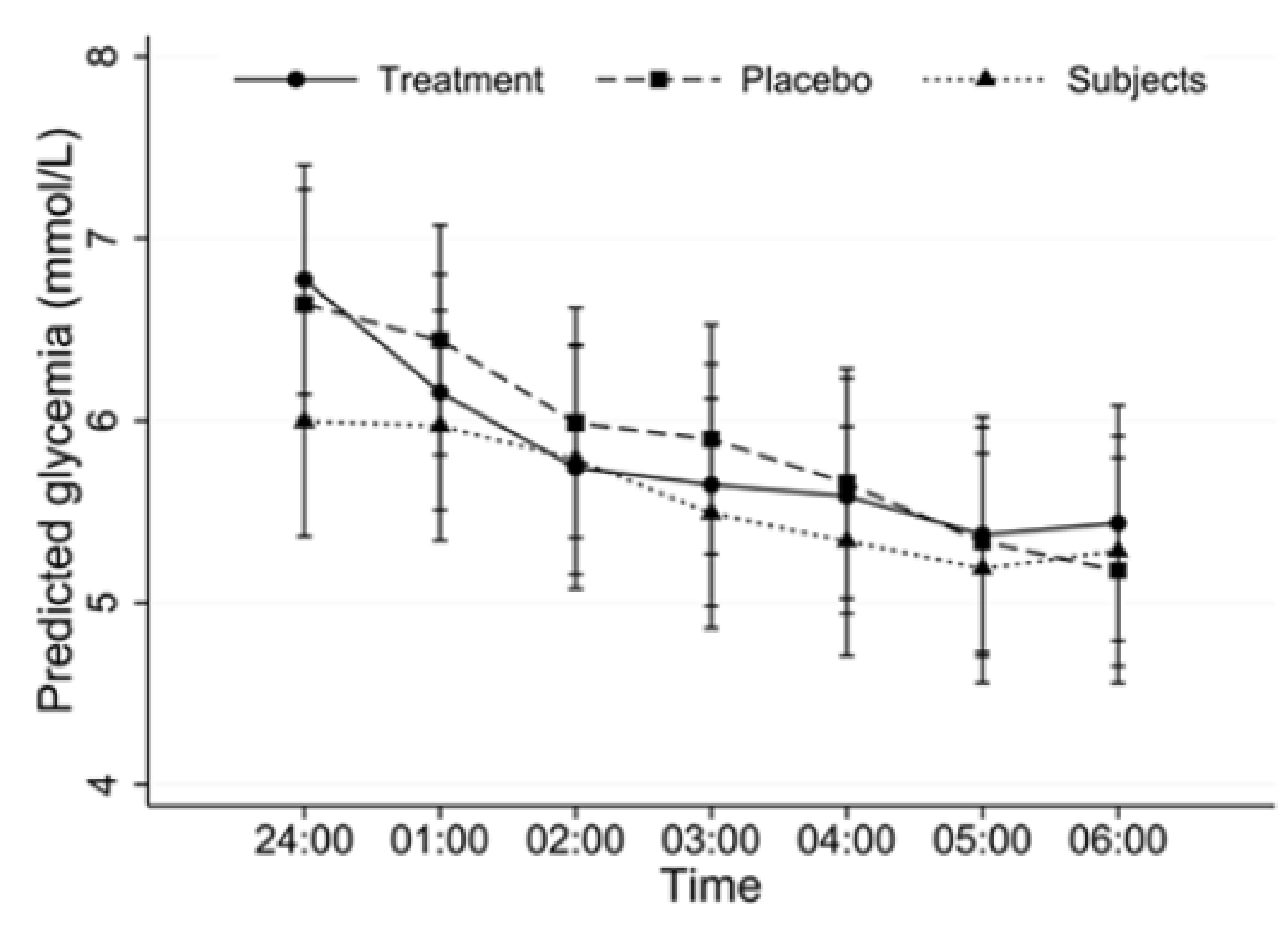

Figure 3. Post-exercise average levels of glycemia followed up from midnight to 0600 a.m. in patients and healthy subjects $P$ for time-by-group interaction was 0.80 